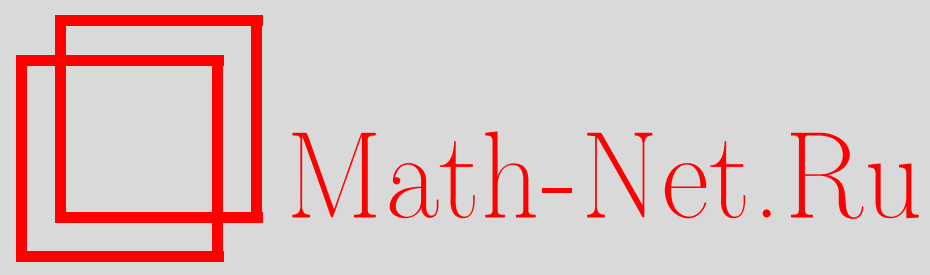

В. Н. Деменко, О базисах в весовых пространствах Соболева, Матем. заметки, 2000, том 67, выпуск 3, 343-354

DOI: https://doi.org/10.4213/mzm847

Использование Общероссийского математического портала Math-Net.Ru подразумевает, что вы прочитали и согласны с пользовательским соглашением http://www. mathnet.ru/rus/agreement

Параметры загрузки:

IP: 54.162 .27 .143

26 апреля 2023 г., 16:03:06

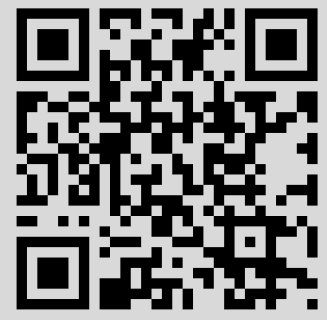




\title{
О БАЗИСАХ В ВЕСОВЫХ ПРОСТРАНСТВАХ СОБОЛЕВА
}

\section{В.Н. Деменко}

\begin{abstract}
В статье строится система гладких двумерных сплайнов, а также описывается класс мер, при которых эта система будет базисом в весовом пространстве Соболева на квадрате.
\end{abstract}

Библиография: 9 названий.

В статье изучаются сплайн-базисы в весовых пространствах Соболева на квадрате. В 1932 году Банах поставил вопрос о существовании гладкого базиса в пространстве гладких функций на квадрате. Оказалось (Чисельский [1], Шонефельд [2]), что базисом может служить система гладких двумерных сплайнов. В 1972 году Чисельский и Домста [3] построили ортонормированную систему тензорных произведений сплайнов, являющуюся базисом в пространстве $C^{r}\left(I^{d}\right)$ и пространстве Соболева $W_{p}^{r}\left(I^{d}\right)(r \geqslant 0$, $d \geqslant 1, p \geqslant 1, I=[0,1])$. Целью данной работы является построение системы гладких двумерных сплайнов, а также описание класса мер $\mu$, при которых эта система будет базисом в весовом пространстве Соболева на квадрате.

Мы будем рассматривать заданные на квадрате меры $\mu$ с непрерывными весами $\rho(\boldsymbol{t})$, имеющими, быть может, только конечное число нулей $\left\{\boldsymbol{t}^{(j)}\right\}(j=1, \ldots, k)$, такими, что для некоторых констант $\delta>0, c>0, C>0$ и набора $\left\{\alpha_{j}\right\}\left(\alpha_{j}>0\right)$

$$
c\left|\boldsymbol{t}-\boldsymbol{t}^{(j)}\right|^{\alpha_{j}} \leqslant \rho(\boldsymbol{t}) \leqslant C\left|\boldsymbol{t}-\boldsymbol{t}^{(j)}\right|^{\alpha_{j}}, \quad \boldsymbol{t} \in O_{\delta}(\boldsymbol{t}),
$$

где $O_{\delta}(\boldsymbol{t})$ означает $\delta$-окрестность точки $\boldsymbol{t}$.

В работе мы будем иметь дело со следующими функциональными пространствами:

а) пространством $C^{r}\left(I^{2}\right) r$ раз непрерывно дифференцируемых функций на квадрате $I^{2}$ с нормой

$\|f\|_{C^{r}\left(I^{2}\right)}=\max _{|\boldsymbol{a}| \leqslant r}\left(\max _{\boldsymbol{t} \in I^{2}}\left|D^{\boldsymbol{a}} f(\boldsymbol{t})\right|\right), \quad \boldsymbol{a} \in \mathbb{Z}_{+}^{2}, \quad|\boldsymbol{a}|=a_{1}+a_{2}, \quad D^{\boldsymbol{a}}=\frac{\partial^{|\boldsymbol{a}|}}{\partial t_{1}^{a_{1}} \partial t_{2}^{a_{2}}} ;$

б) пространством $L_{2}\left(I^{2}, \rho\right)$ со скалярньм произведением

$$
(f, g)=\int_{I^{2}} f(\boldsymbol{t}) g(\boldsymbol{t}) \rho(\boldsymbol{t}) d \boldsymbol{t}
$$

в) весовым пространством Соболева $W_{p}^{r}\left(I^{2}, \rho\right)=\left\{f \in L_{p}\left(I^{2}, \rho\right):\|f\|_{W_{p}^{r}\left(I^{2}, \rho\right)}<\infty\right\}$, где

$$
\|f\|_{W_{p}^{r}\left(I^{2}, \rho\right)}=\sum_{|\boldsymbol{a}| \leqslant r}\left(\int_{I^{2}}\left|D^{\boldsymbol{a}} f(\boldsymbol{t})\right|^{p} \rho(\boldsymbol{t}) d \boldsymbol{t}\right)^{1 / p} .
$$


Пространство Соболева определяется в терминах обобшенных производных. Если вес $\rho(\boldsymbol{t})$ удовлетворяет условию (1), то пространство $W_{p}^{r}\left(I^{2}, \rho\right)$ является пополнением $C^{r}\left(I^{2}\right)$ по отношению к норме $(2)$.

Основным результатом данной работы является

ТЕОРема 2. Пусть вес $\rho(\boldsymbol{t})$ удовлетворяет условию (1). Тогда для любого $r \geqslant 1$ существует система сплайн-функиий $\left\{f_{n}^{(r, \rho)}(\boldsymbol{t}), n \in \mathbb{N}\right\}$, являющаяся базисом в $W_{p}^{r}\left(I^{2}, \rho\right)$.

Система из теоремы 2 ортогональна в пространстве $L_{2}\left(I^{2}, \rho\right)$. Одномерньй $B$-сплайн с множеством узлов $\theta=\left\{t_{-1}, \ldots, t_{r+1}\right\}, a \leqslant t_{-1} \leqslant \cdots \leqslant t_{r+1} \leqslant b$, определяется равенством

$$
M(t)=M(t \mid \theta)=(r+2)\left[t_{-1}, \ldots, t_{r+1}\right](\cdot-t)_{+}^{r+1},
$$

где $\left[t_{-1}, \ldots, t_{r+1}\right] f-$ разделенная разность функции $f$, a $(\cdot)_{+}^{r}-$ усеченная степень: $x_{+}^{r}=x^{r}$ при $x \geqslant 0$ и $x_{+}^{r}=0$ при $x \leqslant 0$. В случае различных узлов имеет место равенство

$$
M(t \mid \theta)=\sum_{i=-1}^{r+1} \frac{(r+2)\left(t_{i}-t\right)_{+}^{r+1}}{\prod_{\substack{j=-1 \\ j \neq i}}^{r+1}\left(t_{i}-t_{j}\right)}=\sum_{i=-1}^{r+1} \frac{(r+2)\left(t-t_{i}\right)_{+}^{r+1}}{\prod_{\substack{j=0 \\ j \neq i}}^{\left.r+t_{i}-t_{j}\right)}}
$$

Введем семейство двоичных разбиений $\Theta_{n}(n \in \mathbb{N})$ оси $\mathbb{R}:$

$$
\Theta_{n}=\left\{\theta_{n, j}, j \in \mathbb{Z} ; \theta_{n, 0}=0, \theta_{n, n}=1, \theta_{n, j}<\theta_{n, j+1}\right\}
$$

Если $n=1$, то положим $\theta_{n, j}=j(j \in \mathbb{Z})$, а если $n=2^{\lambda}+\nu$, где $\lambda \geqslant 0,1 \leqslant \nu \leqslant 2^{\lambda}$, то

$$
\theta_{n, j}= \begin{cases}\frac{j}{2^{\lambda+1}}, & j=\ldots,-1,0,1, \ldots, 2 \nu \\ \frac{j-\nu}{2^{\lambda}}, & j=2 \nu+1,2 \nu+2, \ldots\end{cases}
$$

Возьмем меру $\mu$ с весом, удовлетворяющим $(1)$, и построим по ней и разбиениям $\Theta_{n}$ еще два семейства разбиений

$$
T_{n, i}=\left\{\tau_{n, i}^{(j)}, j \in \mathbb{Z}\right\}, \quad i=1,2, \quad n \geqslant n_{0}=\frac{16(r+2)^{2}}{\delta^{2}}
$$

$\tau_{n, i}^{(j)}=\left\{\begin{array}{l}t_{i}^{(l)}, \quad \text { если существует } l \text { такое, что } \frac{1}{2}\left(\theta_{n, j-1}+\theta_{n, j}\right)<t_{i}^{(l)} \leqslant \frac{1}{2}\left(\theta_{n, j+1}+\theta_{n, j}\right), \\ \theta_{n, j} \text { в противном случае. }\end{array}\right.$

Обозначим через $I_{n, i}^{(j)}$ интервал $\left(\tau_{n, i}^{(j-1)}, \tau_{n, i}^{(j)}\right)$. Положим

$$
\sigma_{n}= \begin{cases}2 \nu, & \text { если } \nu<2^{\lambda} \\ 0, & \text { если } \nu=2^{\lambda}\end{cases}
$$

Определим по $T_{n, i}$ последовательность $B$-сплайнов

$$
M_{n, j, i}^{(r)}(t)=M\left(t \mid \tau_{n, i}^{(j-1)}, \ldots, \tau_{n, i}^{(j+r+1)}\right)
$$


Они являются сплайнами $(r+1)$-й степени по отношению к разбиению $T_{n, i}$ и имеют, в частности, следующие свойства (см., например, [4]):

1) $M_{n, j, i}^{(r)} \geqslant 0, \operatorname{supp} M_{n, j, i}^{(r)}=\left\langle\tau_{n, i}^{(r)}, \tau_{n, i}^{(j+r+1)}\right\rangle$;

2) $\sum_{j} M_{n, j, i}^{(r)}(t) \equiv 1, t \in[0,1]$;

3) если $p \geqslant 1$, то для нормы $M_{n, j, i}^{(r)}$ в $L_{p}(\mathbb{R})$ справедлива оценка

$$
r^{-1 / q}\left\|M_{n, j, i}^{(r)}\right\|_{1} \leqslant\left\|M_{n, j, i}^{(r)}\right\|_{p} \leqslant\left\|M_{n, j, i}^{(r)}\right\|_{1}^{1 / p}
$$

4) для $\Delta \subset(0,1)$ система нетривиальных ограничений $\left.M_{n, j, i}^{(r)}\right|_{\Delta}$ является базисом в пространстве сплайнов на $\Delta$.

Многомерные $B$-сплайны были определены де Бором в [5]. Детальное описание их свойств, а также свойств многомерных бокс-сплайнов можно найти в [6]. Нам будет удобно пользоваться двумерными сплайнами, определяемьми как произведения одномерных $B$-сплайнов.

Каждое целое $n \geqslant(r+2)^{2}$ может быть представлено единственным образом в виде $n=(l+r+1)(l+r+1+\varepsilon)+m$, где $l \geqslant 1, \varepsilon=0,1,0 \leqslant m \leqslant l+r+\varepsilon$.

Введем подпространства $\Lambda_{n}^{(r)}\left(n \geqslant n_{0}\right)$ пространства $C^{r}\left(I^{2}\right)$ :

$$
\Lambda_{n}^{(r)}= \begin{cases}\operatorname{span}\left[M_{l+\varepsilon, i, 1}^{(r)}\left(x_{1}\right) M_{l, j, 2}^{(r)}\left(x_{2}\right),-r \leqslant i \leqslant l+\varepsilon,-r \leqslant j \leqslant l\right], & \text { если } m=0, \\ \operatorname{span}\left[M_{l, i, 1}^{(r)}\left(x_{1}\right) M_{l, j, 2}^{(r)}\left(x_{2}\right),-r \leqslant i, j \leqslant l ;\right. & \\ \left.M_{l+1, \sigma_{l+1}, 1}^{(r)}\left(x_{1}\right) M_{l, j, 2}^{(r)}\left(x_{2}\right),-r \leqslant j \leqslant m-r-1\right], & \text { если } m>0, \varepsilon=0, \\ \operatorname{span}\left[M_{l+1, i, 1}^{(r)}\left(x_{1}\right) M_{l, j, 2}^{(r)}\left(x_{2}\right),-r \leqslant i \leqslant l+1,-r \leqslant j \leqslant l ;\right. & \\ \left.M_{l+1, i, 1}^{(r)}\left(x_{1}\right) M_{l+1, \sigma_{l+1}, 2}^{(r)}\left(x_{2}\right),-r \leqslant i \leqslant m-r-1\right], & \text { если } m>0, \varepsilon=1 .\end{cases}
$$

Обозначим через $\varphi_{n, j}^{(r)}$ занумерованную произвольным образом систему произведений $B$-сплайнов, участвующих в определении $\Lambda_{n}^{(r)}$. Носитель каждой функции $f$ из $\Lambda_{n}^{(r)}$ состоит из $(r+2)^{2}$ прямоугольников, на каждом из которых $f$ бесконечно дифференцируема. Множество всех этих прямоугольников обозначим через $\Pi_{n}$.

Возьмем область $\Delta \subseteq I^{2}$ и целое $n \geqslant n_{0}$, определим семейства множеств $\Omega_{n}^{(r)}(\Delta)$, $U_{n}^{(r)}(\Delta)$ и операторов $\Phi_{n, \Delta}^{(r)}$ :

$$
\begin{gathered}
\Omega_{n}^{(r)}(\Delta)=\left\{j: \operatorname{supp} \varphi_{n, j}^{(r)} \cap \Delta \neq \varnothing\right\}, \quad U_{n}^{(r)}(\Delta)=\bigcup_{\substack{j \in \Omega_{n}^{(r)}(\Delta) \\
\text { supp }}} \sup \varphi_{n, j}^{(r)}, \\
\Phi_{n, \Delta}^{(r)}: \Lambda_{n}^{(r)} \rightarrow \Lambda_{n}^{(r)}, \quad \Phi_{n, \Delta}^{(r)}\left(\sum_{j=1}^{n} c_{j} \varphi_{n, j}^{(r)}\right)=\sum_{\substack{j=1 \\
j \notin \Omega_{n}^{(r)}(\Delta)}}^{n} c_{j} \varphi_{n, j}^{(r)} .
\end{gathered}
$$

Проведем вертикальные прямые через точки $\left(\tau_{l+\varepsilon, 1}^{(i(r+2))}, 0\right)$ и горизонтальные через точки $\left(0, \tau_{l, 2}^{(j(r+2))}\right)$. Эти прямые разобьют все прямоугольники из $\Pi_{n-m}$ на блоки, состоящие из $(r+2)^{2}$ прямоугольников, если блок не является правым или верхним граничным и, быть может, меньшего числа, если блок граничньй. Обозначим эти блоки 
через $\pi_{i, j}^{(n)}$, где $(i, j)$ - обычная нумерация: $i$ слева направо, $j$ снизу вверх. Пусть $\omega_{n}$ обозначает множество индексов всех блоков $\pi_{i, j}^{(n)}$ из $I^{2}, \Delta_{i, j}^{(n)}-$ прямоугольник из $\Pi_{n-m}$, являющийся левым нижним в $\pi_{i, j}^{(n)}$, а $t_{i, j}^{(n)}$ - левая нижняя вершина $\Delta_{i, j}^{(n)}$. Нетрудно заметить, что носитель произвольной функции $\varphi_{n, m}^{(r)}$ имеет непустое пересечение с одним и только одним из $\Delta_{i, j}^{(n)}$. Определим последовательность операторов

$$
P_{i, j}^{(n)}: \Lambda_{n}^{(r)} \rightarrow \Lambda_{n}^{(r)}, \quad n \geqslant n_{0} ; \quad P_{i, j}^{(n)}\left(\sum_{m=1}^{n} c_{m} \varphi_{n, m}^{(r)}\right)=\sum_{m \in \Omega_{n}^{(r)}\left(\Delta_{i, j}^{(n)}\right)} c_{m} \varphi_{n, m}^{(r)}
$$

Заметим, что для $f \in \Lambda_{n}^{(r)}$

$$
\sum_{(i, j) \in \omega_{n}}\left(P_{i, j}^{(n)} f\right)(\boldsymbol{t})=f(\boldsymbol{t}), \quad \boldsymbol{t} \in \mathbb{R}^{2}
$$

Обозначим для $p \geqslant 1$ и $\alpha \in\left\{\alpha_{1}, \ldots, \alpha_{k}, 0\right\}$ (набор $\alpha$ определен в (1)) через $X_{p, \alpha}$ линейное пространство $\Lambda_{n}^{(r)}$ с нормой $\|f\|_{X_{p, \alpha}}=\|f\|_{L_{p}\left(I^{2},|\boldsymbol{t}|^{\alpha}\right)}$, а через $Y_{p, \alpha}$ то же пространство с нормой $\|f\|_{Y_{p, \alpha}}=\|f\|_{W_{p}^{r}\left(\mathbb{R}^{2},|\boldsymbol{t}|^{\alpha}\right)}$. Положим

$$
A=\max _{\alpha \in\left\{\alpha_{1}, \ldots, \alpha_{k}, 0\right\}}\left\|P_{0,0}^{\left(n_{0}\right)}\right\|_{X_{p, \alpha} \rightarrow Y_{p, \alpha}} .
$$

Ниже через $A$ мы будем обозначать константы, зависящие только от $r$ и $p$, вообще говоря, разные.

Из последнего неравенства с учетом соображений подобия и оценки (1) получаем, что для любых $f \in \Lambda_{n}^{(r)}\left(n \geqslant n_{0}\right)$ и $(i, j) \in \omega_{n}$

$$
\left\|P_{i, j}^{(n)} f\right\|_{W_{p}^{r}\left(\mathbb{R}^{2}, \rho\right)} \leqslant A n^{r / 2}\|f\|_{L_{p}\left(\Delta_{i, j}^{(n)}, \rho\right)} .
$$

Нам понадобится следующая простая модификация результата де Бора [7, с. 272-273] для двумерных сплайнов.

ЛЕмма 1. Для любого иелого $r \geqslant 1$ найдется константа $D_{r}>1$, не зависящая от п и $p(p \geqslant 1)$, такая, что для любого $\Delta \in \Pi_{n}$

$$
D_{r}^{-1}\left(\sum_{j \in \Omega_{n}^{(r)}(\Delta)}\left|c_{j}\right|^{p}\right)^{1 / p} \leqslant\left\|\sum_{j} c_{j} \gamma_{n, j}^{(r)} \varphi_{n, j}^{(r)}\right\|_{L_{p}(\Delta)} \leqslant\left(\sum_{j \in \Omega_{n}^{(r)}(\Delta)}\left|c_{j}\right|^{p}\right)^{1 / p}
$$

əде $\gamma_{n, j}^{(r)}=\left\|\varphi_{n, j}^{(r)}\right\|_{L_{p}\left(I^{2}\right)}^{-1 / p}$.

Теперь докажем следующую простую лемму.

ЛЕмма 2. Пусть $S \in \Lambda_{n}^{(r)}$ и пусть прямоугольник $\Delta \subseteq I^{2}$. Тогда для любого $\delta \in \Pi_{n}^{(r)}$ такого, что $\delta \in U_{n}^{(r)}(\Delta) \backslash \Delta$,

$$
\left\|\Phi_{n, \Delta}^{(r)} S\right\|_{C(\delta)} \leqslant D_{r}\|S\|_{C(\delta)} .
$$


ДоКАЗАТЕЛЬСТво. Пусть $\delta \in \Pi_{n}^{(r)}$ и $\delta \in U_{n}^{(r)}(\Delta) \backslash \Delta$. Тогда по лемме 1

$$
\begin{gathered}
\|S\|_{C(\delta)}=\left\|\sum_{j=1}^{n} c_{j} \varphi_{n, j}^{(r)}\right\|_{C(\delta)} \geqslant D_{r}^{-1} \max _{j \in \Omega_{n}^{(r)}(\delta)}\left|c_{j}\right|, \\
\left\|\Phi_{n, \Delta}^{(r)}(S)\right\|_{C(\delta)}=\left\|\sum_{\substack{j=1 \\
j \notin \Omega_{n}^{(r)}(\Delta)}}^{n} c_{j} \varphi_{n, j}^{(r)}\right\|_{C(\delta)} \leqslant \max _{j \in \Omega_{n}^{(r)}(\delta) \backslash \Omega_{n}^{(r)}(\Delta)}\left|c_{j}\right| .
\end{gathered}
$$

Комбинация последних двух неравенств дает (4).

Лемма 3. Для любого иелого $r \geqslant 1$ существует последовательность линейных операторов $Q_{n}^{(r)}$ таких, что для $X=W_{p}^{r}\left(I^{2}, \rho\right)$ или $C^{r}\left(I^{2}\right)$ u $Y=L_{p}\left(I^{2}, \rho\right)$ или, соответственно, $C\left(I^{2}\right)$

$$
\begin{gathered}
\left\|Q_{n}^{(r)}\right\|_{X \rightarrow X} \leqslant A, \\
\left\|Q_{n}^{(r)}-E\right\|_{X \rightarrow Y} \leqslant A n^{-r / 2},
\end{gathered}
$$

где $E$ - тождественный оператор, $A$ - константа, зависящая только от $r$ и $p$.

ДокАЗАТЕЛЬСТво. Фиксируем $n \geqslant n_{0}$. Возьмем произвольный прямоугольник $\Delta_{i, j}^{(n)}$ $\left((i, j) \in \omega_{n}\right)$. Обозначим через $T(\boldsymbol{t}, \boldsymbol{x})$ многочлен Тейлора функции $f(\boldsymbol{t})$ в точке $\boldsymbol{x}$ :

$$
T(\boldsymbol{t}, \boldsymbol{x})=\sum_{|\boldsymbol{k}| \leqslant r-1} \frac{1}{k_{1} ! k_{2} !} D^{\boldsymbol{k}} f(\boldsymbol{x})\left(t_{1}-x_{1}\right)^{k_{1}}\left(t_{2}-x_{2}\right)^{k_{2}} .
$$

Введем оператор $Q_{\Delta}$ :

$$
\left(Q_{\Delta} f\right)(\boldsymbol{t})=\frac{1}{\mu(\Delta)} \iint_{\Delta} T(\boldsymbol{t}, \boldsymbol{x}) \rho(\boldsymbol{x}) d \boldsymbol{x} .
$$

Имеем

$$
f(\boldsymbol{t})-\left(Q_{\Delta} f\right)(\boldsymbol{t})=\frac{1}{\mu(\Delta)} \iint_{\Delta}(f(\boldsymbol{t})-T(\boldsymbol{t}, \boldsymbol{x})) \rho(\boldsymbol{x}) d \boldsymbol{x} .
$$

Точки $\boldsymbol{x}$ и $\boldsymbol{t}$ можно соединить, например, такими путями:

$$
\begin{array}{ll}
\Gamma_{1}(\boldsymbol{x}, \boldsymbol{t}): & \left(x_{1}, x_{2}\right)-\left(t_{1}, x_{2}\right)-\left(t_{1}, t_{2}\right), \\
\Gamma_{2}(\boldsymbol{x}, \boldsymbol{t}): & \left(x_{1}, x_{2}\right)-\left(x_{1}, t_{2}\right)-\left(t_{1}, t_{2}\right), \\
\Gamma_{3}(\boldsymbol{x}, \boldsymbol{t}): & \left(x_{1}, x_{2}\right)-\left(t_{2}, x_{2}\right)-\left(t_{2}, t_{2}\right)-\left(t_{1}, t_{2}\right), \\
\Gamma_{4}(\boldsymbol{x}, \boldsymbol{t}): & \left(x_{1}, x_{2}\right)-\left(x_{1}, t_{1}\right)-\left(t_{1}, t_{1}\right)-\left(t_{1}, t_{2}\right) .
\end{array}
$$

Обозначим через $\vartheta_{r}$ множество $\{1,2\}^{r}$. Пусть для $l \in \vartheta_{r} l^{\prime}$ и $l^{\prime \prime}$ обозначают соответственно числа единищ и двоек в наборе $l$. Положим $\boldsymbol{a}_{l}=\left(l^{\prime}, l^{\prime \prime}\right)$. Интегрируя производные функции $f$ по пути $\Gamma_{i}$, получим для разности $f(\boldsymbol{t})-T(\boldsymbol{x}, \boldsymbol{t})$ формулу

$$
f(\boldsymbol{t})-T(\boldsymbol{x}, \boldsymbol{t})=\sum_{l \in \vartheta_{r}} \int_{\Gamma_{i}(\boldsymbol{x}, \boldsymbol{t})} \int_{\Gamma_{i}\left(\boldsymbol{x}, \boldsymbol{u}^{(1)}\right)} \cdots \int_{\Gamma_{i}\left(\boldsymbol{x}, \boldsymbol{u}^{(r-1)}\right)} D^{\boldsymbol{a}_{l}} f\left(\boldsymbol{u}^{(r)}\right) d u_{l_{r}}^{(r)} \cdots d u_{l_{1}}^{(1)}
$$


Возьмем произвольные прямоугольник $\Delta=\Delta_{i, j}^{(n)}\left((i, j) \in \omega_{n}\right)$, точку $\boldsymbol{t} \in I^{2}$, число $\xi \in \mathbb{R}$ и определим для $k=1,2,3,4$ множества

$$
\begin{aligned}
& \delta_{1, k}(\Delta, \boldsymbol{t}, \xi)=\left\{\boldsymbol{x} \in \Delta: \forall \boldsymbol{s} \in \Gamma_{k}(\boldsymbol{x}, \boldsymbol{t}) \quad \xi \rho(\boldsymbol{s}) \geqslant \rho(\boldsymbol{x})\right\} \\
& \delta_{2, k}(\Delta, \boldsymbol{t}, \xi)=\left\{\boldsymbol{x} \in \Delta: \forall s \in \Gamma_{k}(\boldsymbol{x}, \boldsymbol{t}) \quad \xi \rho(\boldsymbol{s}) \geqslant \rho(\boldsymbol{t})\right\} .
\end{aligned}
$$

Пусть $\Delta^{\prime}=d_{1}^{\prime} \times d_{2}^{\prime}=\Delta_{i^{\prime}, j^{\prime}}^{(n)}$, а $\Delta^{\prime \prime}=d_{1}^{\prime \prime} \times d_{2}^{\prime \prime}=\Delta_{i^{\prime \prime}, j^{\prime \prime}}^{(n)}$ или $\Delta^{\prime \prime}=\pi_{i^{\prime \prime}, j^{\prime \prime}}^{(n)}$, где $\left(i^{\prime}, j^{\prime}\right),\left(i^{\prime \prime}, j^{\prime \prime}\right) \in \omega_{n}$ и $0 \leqslant i^{\prime \prime}-i^{\prime} \leqslant 1,0 \leqslant j^{\prime \prime}-j^{\prime} \leqslant 1$.

Для упрощения дальнейшей записи перенесем начало координат в точку $\boldsymbol{t}_{i^{\prime}, j^{\prime}}$, если $\rho(\boldsymbol{t}) \neq 0$ на

$$
\pi=\bigcup_{\substack{0 \leqslant i-i^{\prime} \leqslant 1 \\ 0 \leqslant j-j^{\prime} \leqslant 1}} \pi_{i, j}^{(n)}
$$

или в точку $\boldsymbol{t} \in \pi$, в которой $\rho(\boldsymbol{t})=0$. Обозначим через $\Delta^{*}=d_{1}^{*} \times d_{2}^{*}$ наименьший из квадратов, содержащий $\Delta^{\prime} \cup \Delta^{\prime \prime}$ с левьм нижним концом в нуле, если $\rho\left(\boldsymbol{t}_{i^{\prime}, j^{\prime}}\right)=0$, или с центром в нуле в противном случае. Исходя из свойств веса $\rho$ (формулы $(1)$ и непрерьвности) нетрудно обнаружить, что для любых точек $\boldsymbol{t}, \boldsymbol{x} \in \Delta$ таких, что $|\boldsymbol{t}|<|\boldsymbol{x}|$, и любого прямоугольника $\widetilde{\Delta} \supset \Delta$ будет справедливо $\rho(\boldsymbol{t})<M \rho(\boldsymbol{x}), \rho(\boldsymbol{t})<M \rho_{\Delta}$,

$$
\mu \widetilde{\Delta}<M \mu \Delta \cdot \frac{|\widetilde{\Delta}|^{\alpha}}{|\Delta|^{\alpha}}
$$

где

$$
M=\max \left\{\frac{C}{c}, \sup _{I^{2}} \rho(\boldsymbol{t}) / \inf _{I^{2} \backslash \bigcup_{i} O_{\delta}\left(\boldsymbol{t}^{(i)}\right)} \rho(\boldsymbol{t})\right\}, \quad \alpha=\max \left\{1, \alpha_{1}, \ldots, \alpha_{k}\right\},
$$

$|\Delta|$ - площадь прямоугольника $\Delta$, а $\rho_{\Delta}=\mu \Delta /|\Delta|-$ среднее значение веса $\rho$ на прямоугольнике $\Delta$.

Оценим для $i=1,2, j=1,2,3,4$ интегралы

$$
I_{i, j}=\frac{1}{\mu \Delta^{\prime}}\left\|\iint_{\delta_{i, j}\left(\Delta^{\prime}\right)}(f(\boldsymbol{t})-T(\boldsymbol{x}, \boldsymbol{t})) \rho(\boldsymbol{x}) d \boldsymbol{x}\right\|_{L_{p}\left(\Delta^{\prime \prime}, \rho\right)} .
$$

Имеем

$$
\begin{aligned}
I_{1, j} \leqslant & \frac{\xi}{\mu \Delta^{\prime}} \sum_{l \in \vartheta_{r}} \| \iint_{\delta_{1, j}} \int_{\Gamma_{j}(\boldsymbol{x}, \boldsymbol{t})} \cdots \int_{\Gamma_{j}\left(\boldsymbol{x}, \boldsymbol{u}^{(r-1)}\right)}\left|D^{\boldsymbol{a}_{l}} f\left(\boldsymbol{u}^{(r)}\right)\right| \\
& \times \rho\left(\boldsymbol{u}^{(r)}\right) d u_{l_{r}}^{(r)} \cdots d u_{l_{1}}^{(1)} d \boldsymbol{x} \|_{L_{p}\left(\Delta^{\prime \prime}, \rho\right)} \\
\leqslant & \frac{\xi 3^{r}|\Delta|^{r / 2}}{\mu \Delta^{\prime}} \sum_{l \in \vartheta_{r}}\left\|\iint_{\Delta^{*}}\left|D^{\boldsymbol{a}_{l}} f(\boldsymbol{u})\right| \rho^{1 / p}(\boldsymbol{u}) \rho^{1 / q}(\boldsymbol{u}) d \boldsymbol{u}\right\|_{L_{p}\left(\Delta^{\prime \prime}, \rho\right)} \\
\leqslant & \frac{A\left|\Delta^{*}\right|^{r / 2}}{\mu \Delta^{\prime}}\left(\mu \Delta^{\prime \prime}\right)^{1 / p}\left(\mu \Delta^{*}\right)^{1 / q} \sum_{l \in \vartheta_{r}}\left(\iint_{\Delta^{*}}\left|D^{\boldsymbol{a}_{l}} f(\boldsymbol{u})\right|^{p} \rho(\boldsymbol{u}) d \boldsymbol{u}\right)^{1 / p} \\
\leqslant & A\left|\Delta^{*}\right|^{r / 2}\|f\|_{W_{p}^{r}\left(\Delta^{*}, \rho\right)}
\end{aligned}
$$


Аналогично оцениваем $I_{2, j}$ :

$$
\begin{aligned}
I_{2, j} \leqslant & \frac{\xi^{1 / p}}{\mu \Delta^{\prime}} \sum_{l \in \vartheta_{r}}\left(\iint_{\Delta^{\prime \prime}}\left|\iint_{\delta_{2, j}} \int_{\Gamma_{j}(\boldsymbol{x}, \boldsymbol{t})} \cdots \int_{\Gamma_{j}\left(\boldsymbol{x}, \boldsymbol{u}^{(r-1)}\right)}\right| D^{\boldsymbol{a}_{l}} f\left(\boldsymbol{u}^{(r)}\right) \mid\right. \\
& \left.\times\left.\rho^{1 / p}\left(\boldsymbol{u}^{(r)}\right) d u_{l_{r}}^{(r)} \cdots d u_{l_{1}}^{(1)} \rho(\boldsymbol{x}) d \boldsymbol{x}\right|^{p} d \boldsymbol{t}\right)^{1 / p} \\
\leqslant & \frac{A}{\left|\Delta^{\prime}\right|} \sum_{l \in \vartheta_{r}}\left|\Delta^{*}\right|^{(r+1) / 2+1 /(2 p)}\left(\int_{d_{1}^{*}}\left(\int_{d_{2}^{*}}\left|D^{\boldsymbol{a}_{l}} f\left(t_{1}, u\right)\right| \rho^{1 / p}\left(t_{1}, u\right) d u\right)^{p} d t_{1}\right)^{1 / p} \\
& +\left(\int_{d_{2}^{*}}\left(\int_{d_{1}^{*}}\left|D^{\boldsymbol{a}_{l}} f\left(u, t_{2}\right)\right| \rho^{1 / p}\left(u, t_{2}\right) d u\right)^{p} d t_{2}\right)^{1 / p} \\
& \left.+\left|\Delta^{*}\right|^{-1 /(2 q)} \iint_{\Delta^{*}}\left|D^{\boldsymbol{a}_{l}} f(\boldsymbol{u})\right| \rho^{1 / p}(\boldsymbol{u}) d \boldsymbol{u}\right)^{1 / \Delta^{\prime}} \\
\leqslant & A\left|\Delta^{*}\right|^{r / 2}\|f\|_{W_{p}^{r}\left(\Delta^{*}, \rho\right)} .
\end{aligned}
$$

Учитьвая свойства веса $\rho$, замечаем, что для любой точки $\boldsymbol{t}$ из $\Delta^{*}$ будет справедливо вложение

$$
\Delta^{\prime} \subset \bigcap_{\substack{i=1,2 \\ j=1,2,3,4}} \delta_{i, j}\left(\Delta^{\prime}, t, M\right) .
$$

Таким образом, получаем

$$
\begin{aligned}
\left\|f-Q_{\Delta^{\prime}} f\right\|_{L_{p}\left(\Delta^{\prime \prime}, \rho\right)} & \leqslant \frac{1}{\mu \Delta^{\prime}} \sum_{\substack{i=1,2 \\
j=1,2,3,4}}\left\|\iint_{\delta_{i, j}}(f(\boldsymbol{t})-T(\boldsymbol{x}, \boldsymbol{t})) \rho(\boldsymbol{x}) d \boldsymbol{x}\right\|_{L_{p}\left(\Delta^{\prime \prime}, \rho\right)} \\
& \leqslant A\|f\|_{W_{p}^{r}\left(\Delta^{*}, \rho\right)} \cdot n^{-r / 2},
\end{aligned}
$$

т.е.

$$
\left\|f-Q_{\Delta^{\prime}} f\right\|_{L_{p}\left(\Delta^{\prime \prime}, \rho\right)} \leqslant\|f\|_{W_{p}^{r}\left(\Delta^{*}, \rho\right)} \cdot n^{-r / 2},
$$

где $A$ - константа, зависящая только от $\rho$ и $r$.

Положим

$$
Q_{n}^{(r)}=\sum_{(i, j) \in \omega_{n}} P_{i, j}^{(n)} Q_{\Delta_{i, j}^{(n)}} .
$$

Теперь выберем произвольную пару индексов $\left(i_{0}, j_{0}\right)$ и оценим, используя $(3)$, норму $\left\|Q_{n}^{(r)} f\right\|_{W_{p}^{r}\left(\pi_{i_{0}, j_{0}}, \rho\right)}$ (для упрощения записи опустим ниже индексы $n$ и $\rho$ ):

$$
\begin{aligned}
\left\|Q^{(r)} f\right\|_{W_{p}^{r}\left(\pi_{i_{0}, j_{0}}\right) \leqslant} & \sum_{\substack{0 \leqslant i-i_{0} \leqslant 1 \\
0 \leqslant j-j_{0} \leqslant 1}}\left\|P_{i, j}\left(Q_{\Delta_{i, j}}^{(r)}-Q_{\Delta_{i_{0}, j_{0}}}^{(r)}\right) f\right\|_{W_{p}^{r}\left(\pi_{i_{0}, j_{0}}\right)} \\
& +\left\|Q_{\Delta_{i_{0}, j 0}}^{(r)} f\right\|_{W_{p}^{r}\left(\pi_{i_{0}, j_{0}}\right)} \\
\leqslant & A n^{r / 2} \sum_{\substack{0 \leqslant i-i_{0} \leqslant 1 \\
0 \leqslant j-j_{0} \leqslant 1}}\left\|\left(Q_{\Delta_{i, j}}^{(r)}-Q_{\Delta_{i_{0}, j_{0}}}^{(r)}\right) f\right\|_{L_{p}\left(\Delta_{i, j}\right)} \\
& +\left\|Q_{\Delta_{i_{0}, j_{0}}}^{(r)} f\right\|_{W_{p}^{r}\left(\pi_{i_{0}, j_{0}}\right) .}
\end{aligned}
$$


Каж дое из слагаемых правой части последнего неравенства оценим отдельно:

$$
\left\|\left(Q_{\Delta_{i, j}}^{(r)}-Q_{\Delta_{i_{0}, j_{0}}}^{(r)}\right) f\right\|_{L_{p}\left(\Delta_{i, j}\right)} \leqslant\left\|Q_{\Delta_{i, j}}^{(r)} f-f\right\|_{L_{p}\left(\Delta_{i, j}\right)}+\left\|Q_{\Delta_{i_{0}, j_{0}}}^{(r)} f-f\right\|_{L_{p}\left(\Delta_{i, j}\right)} .
$$

Воспользуемся (6) при $\Delta^{\prime}=\Delta^{\prime \prime}=\Delta_{i, j}^{(n)}$ :

$$
\left\|Q_{\Delta_{i, j}}^{(r)} f-f\right\|_{L_{p}\left(\Delta_{i, j}\right)} \leqslant A n^{-r / 2}\|f\|_{W_{p}^{r}\left(\Delta_{i, j}\right)} .
$$

Если же $\Delta^{\prime}=\Delta_{i_{0}, j_{0}}$, а $\Delta^{\prime \prime}=\Delta_{i, j}$, то можем записать

$$
\left\|Q_{\Delta_{i_{0}, j_{0}}}^{(r)} f-f\right\|_{L_{p}\left(\Delta_{i, j}\right)} \leqslant A n^{-r / 2}\|f\|_{W_{p}^{r}\left(\Delta_{i_{0}, j_{0}}^{*}\right)},
$$

где

$$
\Delta_{i_{0}, j_{0}}^{*} \subseteq \bigcup_{\substack{\left|i-i_{0}\right| \leqslant 1 \\\left|j-j_{0}\right| \leqslant 1}} \pi_{i, j}
$$

Рассмотрим $\left\|Q_{\Delta_{i, j}}^{(r)} f\right\|_{W_{p}^{r}\left(\pi_{i, j}\right)}$. Так как индексы $i$ и $j$ сейчас меняться не будут, то для упрощения записи опустим их в следующей ниже вькладке. Имеем

$$
\begin{aligned}
\left\|Q_{\Delta}^{(r)} f\right\|_{W_{p}^{r}(\pi)} & \leqslant \sum_{|\boldsymbol{a}| \leqslant r}\left(\int_{\pi}\left|D^{\boldsymbol{a}}\left(\frac{1}{\mu \Delta} \int_{\Delta} T(\boldsymbol{x}, \boldsymbol{t}) \rho(\boldsymbol{x}) d \boldsymbol{x}\right)\right|^{p} \rho(\boldsymbol{t}) d \boldsymbol{t}\right)^{1 / p} \\
& \leqslant(r !)^{2} r^{2}(\mu \pi)^{1 / p}(\mu \Delta)^{-1 / p} \sum_{|\boldsymbol{k}| \leqslant r-1}\left(\int_{\Delta}\left|D^{\boldsymbol{k}} f(\boldsymbol{x})\right|^{p} \rho(\boldsymbol{x}) d \boldsymbol{x}\right)^{1 / p},
\end{aligned}
$$

т.e.

$$
\left\|Q_{\Delta}^{(r)} f\right\|_{W_{p}^{r}(\pi)} \leqslant A\|f\|_{W_{p}^{r}(\Delta)}
$$

Объединяя (9)-(12), получим

$$
\left\|Q_{n}^{(r)} f\right\|_{W_{p}^{r}\left(\pi_{i_{0}, j_{0}}\right)} \leqslant A \sum_{\substack{0 \leqslant i-i_{0} \leqslant 1 \\ 0 \leqslant j-j_{0} \leqslant 1}}\|f\|_{W_{p}^{r}\left(\Delta_{i, j}^{*}\right)}+A\|f\|_{W_{p}^{r}\left(\Delta_{i_{0}, j_{0}}\right)}
$$

или

$$
\left\|Q_{n}^{(r)} f\right\|_{W_{p}^{r}\left(\pi_{i_{0}, j_{0}}\right)} \leqslant A\|f\|_{W_{p}^{r}\left(\Delta_{i_{0}, j_{0}}^{*}\right)}
$$

где $A$ зависит только от $\rho$ и $r$, а $\Delta_{i_{0}}^{*}, j_{0}$ удовлетворяет (11). Воспользуемся (13) для доказательства (5) в случае $X=W_{p}^{r}\left(I^{2}, \rho\right)$. Пусть $f \in W_{p}^{r}\left(I^{2}, \rho\right)$. Тогда (опустим опять в выкладках индекс $\rho$ )

$$
\left\|Q_{n}^{(r)} f\right\|_{W_{p}^{r}\left(I^{2}\right)} \leqslant A\left(\sum_{(i, j) \in \omega_{n}} \sum_{\substack{|k-i| \leqslant 1 \\|l-j| \leqslant 1}}\|f\|_{W_{p}^{r}\left(\pi_{k, l}\right)}^{p}\right)^{1 / p} \leqslant A 9^{1 / p}\|f\|_{W_{p}^{r}\left(I^{2}\right)} .
$$


Теперь докажем (6) для $X=W_{p}^{r}\left(I^{2}, \rho\right)$ и $Y=L_{p}\left(I^{2}, \rho\right)$. С учетом (3) и (8) имеем

$$
\begin{aligned}
& \left\|Q_{n}^{(r)} f-f\right\|_{L_{p}\left(I^{2}\right)}=\left(\sum_{\substack {(i, j) \in \omega_{n} \\
\begin{subarray}{c}{|k-i| \leqslant 1 \\
|l-j| \leqslant 1{ ( i , j ) \in \omega _ { n } \\
\begin{subarray} { c } { | k - i | \leqslant 1 \\
| l - j | \leqslant 1 } }\end{subarray}} \sum_{\substack{|k, l\\
|}}\left(Q_{\Delta_{k, l}}-Q_{\Delta_{i, j}}\right) f+Q_{\Delta_{i, j}} f-f \|_{L_{p}\left(\pi_{i, j}\right)}^{p}\right)^{1 / p} \\
& \quad \leqslant\left(\sum_{(i, j) \in \omega_{n}}\left(A \sum_{\substack{|k-i| \leqslant 1 \\
|l-j| \leqslant 1}}\left\|Q_{\Delta_{k, l}} f-f\right\|_{L_{p}\left(\Delta_{i, j}\right)}+\left\|Q_{\Delta_{i, j}} f-f\right\|_{L_{p}\left(\pi_{i, j}\right)}\right)^{p}\right)^{1 / p} \\
& \quad \leqslant A n^{-r / 2}\|f\|_{W_{p}^{r}\left(I^{2}\right)} .
\end{aligned}
$$

Переходя к пределу при $\rho \rightarrow \infty$ в оценках $\left\|Q_{n}^{(r)} f\right\|_{W_{p}^{r}}$ и $\left\|Q_{n}^{(r)} f-f\right\|_{L_{p}}$ для $f \in C^{r}\left(I^{2}\right)$, убеждаемся в справедливости (5), (6) и для случая $X=C^{r}, Y=C$.

ЛЕмма 4. Пусть мера $\mu$ с весом $\rho$ удовлетворяет условию (1), константа $r \geqslant 0$, прямоугольник $\delta \in \Pi_{n}\left(n \geqslant n_{0}\right)$, а функиия $S \in \Lambda_{n}^{r}$. Тогда для некоторой константы $\beta$, зависящей только от $r u \mu, u$ для любого $p \geqslant 1$

$$
\|S\|_{L_{p}(\delta, \rho)} \geqslant \frac{1}{2}\|S\|_{C(\delta)}(\beta \mu \delta)^{1 / p}
$$

ДоКАЗАТЕЛЬСТво. Для любого полинома $p(t)$ степени $r+1$ на интервале $\tau \subset \mathbb{R}$

$$
\left\|p^{\prime}\right\|_{C(\tau)} \leqslant \frac{2(r+1)^{2}\|p\|_{C(\tau)}}{|\tau|}
$$

(неравенство Маркова, см. [8, с. 323]). Из (15) видим, что существует прямоугольник $\delta^{\prime} \in \Pi_{n}$ такой, что $\delta^{\prime} \subset \delta,\left|\delta^{\prime}\right|>|\delta| /\left(16(r+1)^{4}\right)$ и $\|S(\boldsymbol{t})\| \geqslant \frac{1}{2}\|S\|_{C(\delta)}$ для $\boldsymbol{t} \in \delta^{\prime}$. Тогда с учетом (7)

$$
\|S\|_{L_{p}(\delta, \rho)}>\frac{1}{2}\|S\|_{C(\delta)}\left(\mu \delta^{\prime}\right)^{1 / p}>\frac{1}{2}\|S\|_{C(\delta)}(\beta \mu \delta)^{1 / p}
$$

Лемма 4 доказана.

Обозначим через $\mathbf{B}_{n}^{(r, \rho)} n$-мерное подпространство $L_{p}\left(I^{2}, \rho\right)$ с базисом $\varphi_{n, j}^{(r)}(j=1$, $\ldots, n)$, а через $P_{n}^{(r, \rho)}$ ортогональный проектор пространства $L_{p}\left(I^{2}, \rho\right)$ на подпространство $\mathbf{B}_{n}^{(r, \rho)}$.

ТЕОрема 1. Пусть мера $\mu$ с весом $\rho$ удовлетворяет условию (1). Тогда для любой функиии $f \in C\left(I^{2}\right)$ и любых иелых $n \geqslant 1, r \geqslant 0\left\|P_{n}^{(r, \rho)} f\right\|_{C}<M_{1}\|f\|_{C}$, әде $M_{1}$ - константа, зависящая только от $\alpha=\max _{1 \leqslant j \leqslant k}\left\{\alpha_{j}\right\} u r$.

ДокАЗАТЕЛЬСТво. Выберем $M_{1}$ так, чтобы для всех $l \in \mathbb{Z}_{+}$

$$
\sqrt{M}(2(r+1)(l+1)+1)^{\alpha}<\gamma^{2}\left(1+\gamma^{2}\right)^{1 / 2} M_{1}, \quad M_{1} \beta^{1 / 2}>4
$$

где

$$
\gamma=\min \left\{\frac{1}{8} \beta^{1 / 2} D_{r}^{-1}, \frac{1}{2}\right\}, \quad \beta=M\left(16(r+1)^{4}\right)^{-\alpha}
$$


Для простоты записи функцию $\left(P_{n}^{(r, \rho)} f\right)(\boldsymbol{t})$ обозначим через $S_{n}(\boldsymbol{t})$. Предположим, что для некоторого номера $n$ в некоторой точке $\boldsymbol{t}_{0}\left(\boldsymbol{t}_{0} \in \Delta \subset \Pi_{n}^{r}\right)$ будет вьполнено

$$
\left|S_{n}\left(\boldsymbol{t}_{0}\right)\right|>M_{1}\|f\|_{C\left(I^{2}\right)}
$$

Введем последовательность областей $\Delta_{0}=\Delta, \Delta_{k+1}=U_{n}^{(r)}\left(\Delta_{k}\right) \cap I^{2}$. Покажем, что

$$
\left\|S_{n}-f\right\|_{L_{2}\left(\Delta_{k}, \rho\right)}>H_{k}=\left(1+\gamma^{2}\right)^{k / 2} H_{0}
$$

где $k \in \mathbb{Z}_{+}, H_{0}=\gamma M_{1}\|f\|_{C\left(I^{2}\right)}\left(\mu \Delta_{0}\right)^{1 / 2}$. В самом деле, для $k=0$, учитьвая $(14)$ и $(16)$, получаем

$$
\begin{aligned}
\left\|S_{n}-f\right\|_{L_{2}\left(\Delta_{0}, \rho\right)} & \geqslant\left\|S_{n}\right\|_{L_{2}\left(\Delta_{0}, \rho\right)}-\|f\|_{L_{2}\left(\Delta_{0}, \rho\right)} \\
& >\frac{1}{2} M_{1}\|f\|_{C\left(I^{2}\right)}\left(\beta \mu \Delta_{0}\right)^{1 / 2}-\|f\|_{C\left(I^{2}\right)}\left(\mu \Delta_{0}\right)^{1 / 2} \\
& >\frac{1}{4} M\|f\|_{C\left(I^{2}\right)}\left(\mu \Delta_{0} \beta\right)^{1 / 2}
\end{aligned}
$$

Предположим, что (18) доказано для $k=l$. Возьмем функцию $\widetilde{S}_{n}=\Phi_{n, \Delta_{l}}^{(r)} S_{n}$. Имеем

$$
\widetilde{S}(\boldsymbol{t})= \begin{cases}0, & \text { если } \boldsymbol{t} \in \Delta_{l}, \\ S_{n}(\boldsymbol{t}), & \text { если } \boldsymbol{t} \notin \Delta_{l+1} .\end{cases}
$$

Так как $S_{n}$ - проекция $f$ на $\mathbf{B}_{n}^{(r, \rho)}$, учитьвая $(19)$, получаем

$$
\left\|\widetilde{S}_{n}-f\right\|_{L_{2}\left(\Delta_{l+1}, \rho\right)}>\left\|S_{n}-f\right\|_{L_{2}\left(\Delta_{l+1}, \rho\right)},
$$

т.e.

$$
\left\|\widetilde{S}_{n}\right\|_{L_{2}\left(\Delta_{l+1} \backslash \Delta_{l}, \rho\right)}+\|f\|_{L_{2}\left(\Delta_{l+1}, \rho\right)}>\left\|S_{n}-f\right\|_{L_{2}\left(\Delta_{l}, \rho\right)} .
$$

Поскольку $\left|\Delta_{l+1}\right|<(2(r+1)(l+1)+1)^{2}\left|\Delta_{0}\right|$ и справедливо $(7)$, имеет место

$$
\|f\|_{L_{2}\left(\Delta_{l+1}, \rho\right)} \leqslant\|f\|_{C\left(I^{2}\right)} M^{1 / 2}(2(r+1)(l+1)+1)^{\alpha}\left(\mu \Delta_{0}\right)^{1 / 2},
$$

откуда с учетом (16) и (18) получаем

$$
\|f\|_{L_{2}\left(\Delta_{l+1}, \rho\right)}<\gamma\left(1+\gamma^{2}\right)^{1 / 2} H_{0}<\gamma\left\|S_{n}-f\right\|_{L_{2}\left(\Delta_{l}, \rho\right)} .
$$

Следовательно,

$$
\left\|\widetilde{S}_{n}\right\|_{L_{2}\left(\Delta_{l+1} \backslash \Delta_{l}, \rho\right)}>\left\|S_{n}-f\right\|_{L_{2}\left(\Delta_{l}, \rho\right)}-\|f\|_{L_{2}\left(\Delta_{l+1}, \rho\right)}>(1-\gamma)\left\|S_{n}-f\right\|_{L_{2}\left(\Delta_{l}, \rho\right)} .
$$

Обозначим через $\left\{\delta_{i}\right\}\left(i \in \sigma_{l}\right)$ множество прямоугольников из $\Pi_{m}^{(r)}$ таких, что $\Delta_{l+1} \backslash \Delta_{l}=$ $\bigcup_{i \in \sigma_{l}} \delta_{i}$ и $\left|\delta_{i} \cap \delta_{j}\right|=0$ при $i \neq j$. Используя леммы 2 и 4 , получаем

$$
\begin{aligned}
\left\|\widetilde{S}_{n}\right\|_{L_{2}\left(\Delta_{l+1} \backslash \Delta_{l}, \rho\right)} & <\left(\sum_{i \in \sigma_{l}}\left\|\widetilde{S}_{n}\right\|_{C\left(\delta_{i}\right)}^{2} \mu \delta_{i}\right)^{1 / 2}<D_{r}\left(\sum_{i \in \sigma_{l}}\left\|S_{n}\right\|_{C\left(\delta_{i}\right)}^{2} \mu \delta_{i}\right)^{1 / 2} \\
& <2 D_{r} \beta^{-1 / 2}\left\|S_{n}\right\|_{L_{2}\left(\Delta_{l+1} \backslash \Delta_{l}, \rho\right)}
\end{aligned}
$$


Из двух последних оценок заключаем, что

$$
\left\|S_{n}\right\|_{L_{2}\left(\Delta_{l+1} \backslash \Delta_{l}, \rho\right)}>2 \gamma\left\|S_{n}-f\right\|_{L_{2}\left(\Delta_{l}, \rho\right)} .
$$

Комбинация последнего неравенства с оценкой (20) дает

$$
\left\|S_{n}-f\right\|_{L_{2}\left(\Delta_{l+1} \backslash \Delta_{l}, \rho\right)}>\left\|S_{n}\right\|_{L_{2}\left(\Delta_{l+1} \backslash \Delta_{l}, \rho\right)}-\|f\|_{L_{2}\left(\Delta_{l+1}, \rho\right)}>\gamma\left\|S_{n}-f\right\|_{L_{2}\left(\Delta_{l}, \rho\right)}
$$

откуда

$$
\left\|S_{n}-f\right\|_{L_{2}\left(\Delta_{l+1}, \rho\right)}>\left\|S_{n}-f\right\|_{L_{2}\left(\Delta_{l}, \rho\right)}\left(1+\gamma^{2}\right)^{1 / 2}>H_{l+1} .
$$

Начиная с некоторого номера $l_{0}$ будем иметь $\Delta_{l}=I^{2}\left(l \geqslant l_{0}\right)$, но в этом случае (18) противоречит тому факту, что $S_{n}$-проекция $f$ на $\mathbf{B}_{n}^{(r, \rho)}$. Следовательно, неравенство (17) не может иметь места.

Теорема 1 доказана.

Теорема 1 означает, что система $\left\{f_{n}^{(r, \rho)}\right\}$ будет базисом в пространстве $C\left(I^{2}\right)$. Из этого факта вытекает (см. [9, с. 100]), что она будет базисом и во всех пространствах $L_{p}\left(I^{2}, \rho\right)(\rho \geqslant 1)$.

ТЕОрема 2. Пусть вес $\rho(\boldsymbol{t})$ удовлетворяет условию (1). Тогда для любого $r \geqslant 1$ существует система сплайн-функиий $\left\{f_{n}^{(r, \rho)}(\boldsymbol{t}), n \in \mathbb{N}\right\}$, являющаяся базисом в $W_{p}^{r}\left(I^{2}, \rho\right)$.

ДоКАЗАТЕЛЬСТво. Искомую систему сплайнов определим следующим образом: $f_{n}^{(r, \rho)}$ образуют ортонормированную систему в $L_{2}\left(I^{2}, \rho\right)$ и $f_{1}^{(r, \rho)} \equiv 1$,

$$
f_{n}^{(r, \rho)} \in \begin{cases}\mathbf{B}_{(r+2)^{2}}^{(r, \rho)} & \text { для } n \leqslant n_{0}, \\ \mathbf{B}_{n}^{(r, \rho)} & \text { для } n>n_{0} .\end{cases}
$$

Пусть даны функция $f \in W_{p}^{r}\left(I^{2}, \rho\right)$ и $n_{1} \geqslant n_{0}$. Возьмем оператор $Q_{n_{1}}^{(r)}$, определенньй в лемме 3 , и положим

$$
q(\boldsymbol{t})=\left(Q_{n_{1}}^{(r)} f\right)(\boldsymbol{t})
$$

Рассмотрим сплайн $\varphi(x, y) \in \Lambda_{n}^{(r)}$. Так как для фиксированного $x_{1}^{*} \in I$ (соответственно $\left.x_{2}^{*} \in I\right)$ функция $\varphi\left(x_{1}^{*}, x_{2}\right)$ (соответственно $\varphi\left(x_{1}, x_{2}^{*}\right)$ ) является одномерным сплайном степени $r+1$ на $I$ с интервалами бесконечной гладкости не менъшими $1 /(2 \sqrt{n})$, то, учитывая неравенство Маркова и (14), видим, что

$$
\begin{aligned}
\left\|D^{\boldsymbol{a}} \varphi\right\|_{L_{p}\left(I^{2}, \rho\right)} & =\left(\sum_{\Delta \in \Pi_{n}}\left\|D^{\boldsymbol{a}_{\varphi}}\right\|_{L_{p}(\Delta, \rho)}^{p}\right)^{1 / p} \leqslant\left(\sum_{\Delta}\left\|D^{\boldsymbol{a}} \varphi\right\|_{C(\Delta)}^{p} \mu \Delta\right)^{1 / p} \\
& <A n^{r / 2}\left(\sum_{\Delta}\|\varphi\|_{C(\Delta)}^{p} \mu \Delta\right)^{1 / p} \leqslant A n^{r / 2}\|\varphi\|_{L_{p}\left(I^{2}, \rho\right)}
\end{aligned}
$$

т.е.

$$
\|\varphi\|_{W_{p}^{r}\left(I^{2}, \rho\right)} \leqslant A n^{r / 2}\|\varphi\|_{L_{p}\left(I^{2}, \rho\right)} .
$$




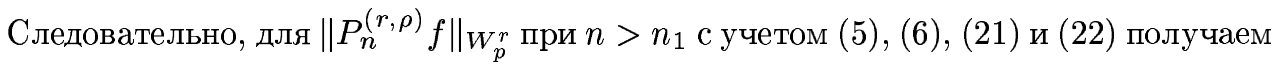

$$
\begin{aligned}
\left\|P_{n}^{(r, \rho)} f\right\|_{W_{p}^{r}\left(I^{2}, \rho\right)} & \leqslant\left\|P_{n}^{(r, \rho)}(f-q)\right\|_{W_{p}^{r}\left(I^{2}, \rho\right)}+\|q\|_{W_{p}^{r}\left(I^{2}, \rho\right)} \\
& <A n^{r / 2}\left\|P_{n}^{(r, \rho)}(f-q)\right\|_{L_{p}\left(I^{2}, \rho\right)}+\|q\|_{W_{p}^{r}\left(I^{2}, \rho\right)} \\
& <A n^{r / 2} M_{1}\|f-q\|_{L_{p}\left(I^{2}, \rho\right)}+A\|f\|_{W_{p}^{r}\left(I^{2}, \rho\right)} \\
& <A\|f\|_{W_{p}^{r}\left(I^{2}, \rho\right)} .
\end{aligned}
$$

Теорема 2 доказана.

\section{СПИСОК ЦИТИРОВАННОЙ ЛИТЕРАТУРЫ}

[1] Ciesielski Z. A construction of basis in $C^{(1)}\left(I^{2}\right) / /$ StudiaMath. 1969. V. 33. № 2. P. 243-247.

[2] Schonefeld S. Schauder bases in spaces of differentiable functions // Bull. Amer. Math. Soc. 1969. V. 75. P. 589-590.

[3] Ciesielski Z., Domsta J. Construction of orthonormal basis in $C^{m}\left(I^{d}\right)$ and $W_{p}^{m}\left(I^{d}\right) / /$ Studia Math. 1972. V. 41. № 2. P. 211-224.

[4] Carry H. B., Schoenberg I. J. On Polya frequence function. IV // J. Anal. Math. 1966. V. 17. P. $71-107$.

[5] de Boor C. Splines as linear combinations of $B$-splines // Approximation Theory. V. II. New York: Acad. Press, 1976. P. 1-47.

[6] Акопян А. А., Саакян А. А. Многомерные сплайны и полиномиальная интерполяция // УМH. 1993. T. 48. № 5. C. 3-76.

[7] de Boor C. The quasi interpolant as a tool in elementary spline theory // Approximation Theory. New York: Acad. Press, 1973. P. 269-276.

[8] Ахиезер Н. И. Лекции по теории аппроксимации. М.: Наука, 1965.

[9] Olevskii A. M. Fourier Series with respect to General Orthogonal Systems. Berlin: Springer, 1975 .

Московский государственный институт электроники и математики

Поступило

04.02 .1998

Исправленный вариант

15.04.1999 